MATHEMATICS OF COMPUTATION

Volume 71, Number 238 , Pages 815-823

S $0025-5718(01) 01396-5$

Article electronically published on September 17,2001

\title{
THE IGUSA LOCAL ZETA FUNCTIONS OF ELLIPTIC CURVES
}

\author{
DIANE MEUSER AND MARGARET ROBINSON
}

\begin{abstract}
We determine the explicit form of the Igusa local zeta function associated to an elliptic curve. The denominator is known to be trivial. Here we determine the possible numerators and classify them according to the KodairaNéron classification of the special fibers of elliptic curves as determined by Tate's algorithm.
\end{abstract}

\section{INTRODUCTION}

In this paper we determine the explicit form of the Igusa local zeta function

$$
Z_{K}(s)=\int_{O_{K}^{(2)}}|f(x, y)|_{K}^{s}|d x d y|_{K},
$$

when $K$ is a local field and $f(x, y)=0$ is the Weierstrass equation of an elliptic curve. The zeta function is determined using a stationary phase formula that was developed by Igusa in 11. We classify the possibilities according to the KodairaNéron classification of the special fibers of elliptic curves as determined by Tate's algorithm 4.

It is known for general polynomial $f$ and char $K=0$ that the Igusa local zeta function is a rational function of $t=q^{-s}$, where $q$ is the cardinality of the residue field and indeed setting $u=q^{-1}$ it is a rational function of $u$ and $t$ with integer coefficients. When $f(x, y)=0$ is nonsingular, it is easily seen that the denominator of the Igusa local zeta function is just $(1-u t)$. For general curves $f(x, y)$ and char $K=0$, the denominator of the Igusa local zeta function has been explicitly determined using the fact that there is an explicit desingularization of the singularities of $f(x, y)=0$ over $K$. This has been done in [2], [5] and shows that the denominator depends upon certain numerical data associated to the exceptional divisors in the resolution. However, the explicit form of the numerator is more difficult to determine and, apart from some specific examples of curves such as those in the form $f(x, y)=x^{m} \pm y^{n}$, has not been determined. Since the cases where $f(x, y)=0$ is an elliptic curve have the simplest possible form of the denominator, it is a natural class of examples in which to study the numerator. We also note that if $P(t)$ is the Poincaré series defined by $P(t)=\sum_{e=0}^{\infty} N_{e} q^{-n e} t^{e}$ with $N_{e}=\operatorname{Card}\left\{x\left(\bmod P_{K}^{e}\right) \mid f(x) \equiv 0\left(\bmod P_{K}^{e}\right)\right\}$ and $P_{K}$ the maximal ideal of the ring of integers $O_{K}$ of $K$, there is the relation $P(t)=\left(1-t Z_{K}\right) /(1-t)$. Hence, one can obtain explicit formulas for $N_{e}$ from the explicit form of $Z_{K}$.

Received by the editor July 10, 2000.

2000 Mathematics Subject Classification. Primary 11S40, 11G07.

Key words and phrases. Local zeta functions, elliptic curves.

This work was supported by National Science Foundation Grant No. DMS-9732228.

(C)2001 American Mathematical Society 


\section{Preliminaries}

As mentioned above, $K$ will denote a local field. We let $O_{K}$ denote the ring of integers in $K$ and $P_{K}$ its maximal ideal. We denote the residue field $O_{K} / P_{K}$ by $k$ and $q=$ Card $k$. We let the image of an element $a$ under the canonical homomorphism $O_{K} \rightarrow k$ be denoted by $\bar{a}$. We choose a uniformizer $\pi \in P_{K}-P_{K}^{2}$. Let ||$_{K}$ denote an absolute value on $K$, which is normalized so that $|\pi|_{K}=q^{-1}$. Let $\nu$ be the corresponding valuation on $K$. We write $x=\pi^{\nu(x)} a c(x)$, and let $\overline{a c}(x)$ denote the image of $a c(x)$ in $k$. We denote by $|d x d y|_{K}$ the Haar measure on $O_{K}^{(2)}$ normalized so that the measure of $O_{K}^{(2)}$ is one. The Igusa local zeta function for a curve $f(x, y)$ is

$$
Z_{K}(s)=\int_{O_{K}^{(2)}}|f(x, y)|_{K}^{s}|d x d y|_{K},
$$

where $s \in \mathbf{C}, \operatorname{Re}(s)>0$. Since it is a rational function in $t=q^{-s}$ and $u=q^{-1}$, we will also write $Z_{K}(u, t)$.

In [1] Igusa introduced what he called a stationary phase formula, which can be an effective method for computing the local zeta function. In particular we let $\bar{S}$ denote the set of singular points of $\bar{f}=0 \in k^{(2)}$. For each $\bar{\zeta}=\left(\bar{\zeta}_{1}, \bar{\zeta}_{2}\right) \in \bar{S}$ we choose a point $\zeta=\left(\zeta_{1}, \zeta_{2}\right) \in O_{K}^{(2)}$ which reduces to $\bar{\zeta}$ under the homomorphism $O_{K}^{(2)} \rightarrow k^{(2)}$ and denote the resulting set of points by $S$. Then the formula is

$$
\begin{aligned}
Z_{K}(u, t)=1-N_{0} u^{2} & +u^{2} t\left(N_{0}-S_{0}\right)(1-u) /(1-u t) \\
& +\sum_{\left(\zeta_{1}, \zeta_{2}\right) \in S} \int_{\left(\zeta_{1}+P_{K}\right) \times\left(\zeta_{2}+P_{K}\right)}|f(x, y)|_{K}^{s}|d x d y|_{K},
\end{aligned}
$$

where $N_{0}=\operatorname{Card}\{\bar{f}=0\}$ and $S_{0}=\operatorname{Card} S$.

We shall assume $f(x, y)=0$ is given by a Weierstrass equation $y^{2}+a_{1} x y+a_{3} y-$ $x^{3}-a_{2} x^{2}-a_{4} x-a_{6}=0$, where $a_{i} \in O_{K}$. We let $\bar{f} \in k[x, y]$ denote the image of $f$ obtained by applying the canonical homomorphism $O_{K} \rightarrow k$ to the coefficients of $f$. In the case where $\bar{f}=0$ is nonsingular, Igusa's stationary phase formula gives

$$
Z_{K}(u, t)=1-N_{0} u^{2}+N_{0}(1-u) u^{2} t /(1-u t) .
$$

When $\bar{f}=0$ is singular, since it is in Weierstrass form it has at most one singular point. After changing coordinates we can assume the singularity is at the origin. We note that this change of coordinates does not affect the value of the integral. In the algorithm below we will frequently use this fact and assume the singularity is at the origin. Let $\bar{\zeta}$ denote the singular point and $\zeta=\left(\zeta_{1}, \zeta_{2}\right)$ denote a lifting of $\bar{\zeta}$ to $O_{K}^{(2)}$. Then the stationary phase formula becomes

$$
\begin{aligned}
Z_{K}(u, t)=1-N_{0} u^{2} & +u^{2} t\left(N_{0}-1\right)(1-u) /(1-u t) \\
& +\int_{P_{K}^{2}}\left|f\left(\zeta_{1}+x, \zeta_{2}+y\right)\right|_{K}^{s}|d x d y|_{K} .
\end{aligned}
$$

We change variables so that $\zeta$ is the origin, then set $(x, y)=\left(\pi x_{1}, \pi y_{1}\right)$. We then factor out the highest possible power of $\pi, \pi^{k_{0}}$ for $k_{0}>0$ from $f\left(\pi x_{1}, \pi y_{1}\right)$. Writing $f_{1}\left(x_{1}, y_{1}\right)=\pi^{-k_{0}} f\left(\pi x_{1}, \pi y_{1}\right)$ we then write the first application of the stationary phase formula as

$$
Z_{K}(u, t)=\left(1-N_{0} u^{2}\right)+u^{2} t\left(N_{0}-1\right)(1-u) /(1-u t)+u^{2} t^{k_{0}} \tilde{I}_{1}
$$


with

$$
\tilde{I}_{1}=\int_{O_{K}^{(2)}}\left|f_{1}\right|_{K}^{s}\left|d x_{1} d y_{1}\right|_{K}
$$

The stationary phase formula can be applied again to $\tilde{I}_{1}$. If

$$
\tilde{I}_{i}=\int_{O_{K}^{(2)}}\left|f_{i}\right|_{K}^{s}\left|d x_{i} d y_{i}\right|_{K}
$$

with $\bar{f}_{i}$ for $i \geq 1$ a polynomial in two variables, the procedure is identical to the above with

$$
f_{i+1}\left(x_{i+1}, y_{i+1}\right)=\pi^{-k_{i}} f_{i}\left(\pi x_{i+1}, \pi y_{i+1}\right) .
$$

However at this and subsequent stages it is possible to have $\bar{f}_{i}$ a polynomial in one variable. In that case we proceed as follows. Suppose $\bar{f}_{i}$ is a polynomial in $x$ alone. Change variables so the singularity of $\bar{f}_{i}$ is at the origin, then set $\left(x_{i}, y_{i}\right)=\left(\pi x_{i+1}, y_{i+1}\right)$. We again factor out the highest possible power of $\pi, \pi^{k_{i}}$ for $k_{i}>0$ from $f_{i}\left(\pi x_{i+1}, y_{i+1}\right)$ and write $f_{i+1}\left(x_{i+1}, y_{i+1}\right)=\pi^{-k_{i}} f_{i}\left(\pi x_{i+1}, y_{i+1}\right)$. Then with $N_{i}=\operatorname{Card}\left\{\bar{f}_{i}=0\right\} \in k^{(2)}$ and $S_{i}$ the cardinality of the set of singular points of $\bar{f}_{i}$ in $k^{(2)}$ we have $S_{i}=q$, so the stationary phase formula gives

$$
\tilde{I}_{i}=1-N_{i} u^{2}+u^{2} t\left(N_{i}-u^{-1}\right)(1-u) /(1-u t)+u t^{k_{i}} \tilde{I}_{i+1} .
$$

In the case where $\bar{f}_{i}=0$ is a polynomial in the single variable, $y$ is handled similarly with $f_{i+1}\left(x_{i+1}, y_{i+1}\right)=\pi^{-k_{i}} f_{i}\left(x_{i+1}, \pi y_{i+1}\right)$.

In order to describe the numerator of $Z_{K}(u, t)$, we let $\tilde{I}_{0}$ denote the original defining integral, $f_{0}=f$ and $P_{i}=P_{i}(u, t)$ the contribution to the numerator of $\tilde{I}_{i}$ from the first two terms of the stationary phase formula applied to $\tilde{I}_{i}$, i.e.,

$$
\begin{aligned}
P_{i} & =\left(1-N_{i} u^{2}\right)(1-u t)+u^{2} t\left(N_{i}-S_{i}\right)(1-u) \\
& =\left(1-N_{i} u^{2}\right)+\left(-u+\left(N_{i}-S_{i}\right) u^{2}+S_{i} u^{3}\right) t,
\end{aligned}
$$

and let $Q$ denote the contribution to the numerator of $\tilde{I}_{i+1}$ when $\bar{f}_{i+1}=0$ is nonsingular, i.e.,

$$
\begin{aligned}
Q & =\left(1-N_{i+1} u^{2}\right)(1-u t)+u^{2} t N_{i+1}(1-u) \\
& =\left(1-N_{i+1} u^{2}\right)+\left(-u+N_{i+1} u^{2}\right) t
\end{aligned}
$$

With the above notation subsequent applications of the stationary phase formula are written as

$$
\tilde{I}_{i}=P_{i}(u, t) /(1-u t)+u^{\delta_{i}} t^{k_{i}} \tilde{I}_{i+1},
$$

when $\bar{f}_{i+1}=0$ is singular with $\delta_{i}$ the number of variables of $\bar{f}_{i}, N_{i}=\operatorname{Card}\left\{\bar{f}_{i}=0\right\}$ and $S_{i}$ the cardinality of the set of singular points of $\bar{f}_{i}$ where both of these are counted in $k^{(2)}$. When $\bar{f}_{i+1}=0$ is nonsingular, the algorithm terminates with

$$
\tilde{I}_{i}=P_{i}(u, t) /(1-u t)+u^{\delta_{i}} t^{k_{i}} Q(u, t) /(1-u t) .
$$

Using the above formulas and notation, we can explicitly determine the zeta function of any elliptic curve $f(x, y)=0$ in Weierstrass form and classify the numerators according to the Kodaira-Néron classification of the special fibers of elliptic curves as determined by Tate's algorithm [4]. In fact the application of the above stationary phase formula closely follows that in Tate's algorithm (see for instance the description of this algorithm given in [3]). In this paper we explicitly describe the process that determines the numerators for the two infinite families $I_{n}$ and $I_{n}^{*}$. 
All of the other cases in Tate's algorithm corresponding to the Kodaira-Néron classification of the special fibers are similarly determined after a finite number of applications of the stationary phase formula and are listed in the final section. We wish to point out that we were assisted with these calculations by the students Mariana Campbell, Ed DuBois, Michael Joyce, Anushka Krishnachander, Kim Schneider, and Jason Slemons who participated in the 1999 Mount Holyoke Number Theory Research Experience for Undergraduates.

\section{THE DETERMinAtion OF THE LOCAL ZETA FUNCTION FOR THE CASES $I_{n}$}

In the $I_{0}$ case, $\nu(\Delta)=0$, where $\Delta$ is the discriminant of $f$. Thus $\bar{f}$ is nonsingular and a single application of the stationary phase formula gives the numerator in the $I_{0}$ case is

$$
\left(1-N_{0} u^{2}\right)+\left(-u+N_{0} u^{2}\right) t
$$

where $N_{0}=\operatorname{Card}\{\bar{f}=0\}$.

To explain the algorithm in the cases $n>0$ we recall the definitions of the following quantities associated to the Weierstrass equation of an elliptic curve:

$$
\begin{aligned}
b_{2} & =a_{1}^{2}+4 a_{2}, \\
b_{4} & =a_{1} a_{3}+2 a_{4}, \\
b_{6} & =a_{3}^{2}+4 a_{6}, \\
b_{8} & =a_{1}^{2} a_{6}+4 a_{2} a_{6}-a_{1} a_{3} a_{4}+a_{2} a_{3}^{2}-a_{4}^{2} \\
& =\left(b_{2} b_{6}-b_{4}^{2}\right) / 4, \\
\Delta & =-b_{2}^{2} b_{8}-8 b_{4}^{3}-27 b_{6}^{2}+9 b_{2} b_{4} b_{6} .
\end{aligned}
$$

For our purposes it is also important to note that $\Delta$ remains invariant under changes of coordinates $x=x^{\prime}+r, y=y^{\prime}+s$. The polynomial $f_{i}$ will have form $f_{i}=$ $c_{1} y_{i}^{2}+a_{1}^{(i)} x_{i} y_{i}+a_{3}^{(i)} y_{i}-c_{2} x_{i}^{3}-a_{2}^{(i)} x^{2}-a_{4}^{(i)} x_{1}-a_{6}^{(i)}$ and we let $b_{8}^{(i)}$ be the abovedefined $b_{8}$ with $a_{k}$ replaced by $a_{k}^{(i)}$.

When $\bar{f}$ has a singularity, we can assume it is at the origin. Then $\pi\left|a_{3}, \pi\right| a_{4}$ and $\pi \mid a_{6}$. In the $I_{n}$ case $\nu\left(b_{2}\right)=0$ and $n=\nu(\Delta)$. When char $k \neq 2$ by making the change of variables replacing $y$ by $\left(y-\bar{a}_{1} x\right) / 2$, one can also see that

$$
N_{0}=\left\{\begin{array}{lll}
q-1 & \text { if } \bar{b}_{2} \in k^{2} \\
q+1 & \text { if } \bar{b}_{2} \notin k^{2}
\end{array}\right.
$$

We shall use the notation $a_{i, j}=\pi^{-j} a_{i}$ as in Tate's algorithm.

If $\nu\left(a_{6}\right)=1$, the first application of the stationary phase formula gives

$$
Z_{K}(u, t)=P_{0} /(1-u t)+u^{2} t \tilde{I}_{1}
$$

with

$$
P_{0}=\left(1-N_{0} u^{2}\right)+\left(-u+\left(N_{0}-1\right) u^{2}+u^{3}\right) t
$$

and $f_{1}=\pi y_{1}^{2}+a_{1} \pi x_{1} y_{1}+a_{3} y_{1}-\pi^{2} x_{1}^{3}-a_{2} \pi x_{1}^{2}-a_{4} x_{1}-a_{6,1}$. Then $\bar{f}_{1}=-\bar{a}_{6,1}$. Using the above formulas combined with our assumptions on $\nu\left(a_{3}\right), \nu\left(a_{4}\right), \nu\left(a_{6}\right)$ and $\nu\left(b_{2}\right)$ we have that $\nu\left(a_{6}\right)=1$ is equivalent to $\nu\left(b_{8}\right)=1$, which in turn is equivalent to $\nu(\Delta)=1$. We have $N_{1}=0$, hence $Q=(1-u t)$. For the remainder of this 
section we let $Q_{1}=u^{2} t(1-u t)$. Thus in the $I_{1}$ case we have that the numerator of $Z_{K}(u, t)$ equals

$$
P_{0}+Q_{1}=\left(1-N_{0} u^{2}\right)+\left(-u+N_{0} u^{2}+u^{3}\right) t-u^{3} t^{2} .
$$

If $n>1$, then $\nu\left(a_{6}\right)>1$ and the first application of the stationary phase formula gives

$$
Z_{K}(u, t)=P_{0} /(1-u t)+u^{2} t^{2} \tilde{I}_{1}
$$

with $f_{1}=y_{1}^{2}+a_{1} x_{1} y_{1}+a_{3,1} y_{1}-\pi x_{1}^{3}-a_{2} x_{1}^{2}-a_{4,1} x_{1}-a_{6,2}$. We have that $\bar{f}_{1}$ is nonsingular if and only if $\nu\left(b_{8}^{(1)}\right)=0$. But one easily sees that $\nu\left(b_{8}^{(1)}\right)=\nu\left(b_{8}\right)-2$. With our current assumptions one sees $\nu(\Delta)=\nu\left(b_{8}\right)$; hence, when $\bar{f}_{1}$ is nonsingular we are in the $I_{2}$ case. We now let $\bar{f}_{1}^{(n s)}$ denote $\bar{f}_{1}$ in this case and $N_{1}^{(n s)}$ denote Card $\left\{\bar{f}_{1}^{(n s)}=0\right\}$. Then

$$
\begin{aligned}
Q & =\left(1-N_{1}^{(n s)} u^{2}\right)(1-u t)+u^{2} t N_{1}^{(n s)}(1-u) \\
& =\left(1-N_{1}^{(n s)} u^{2}\right)+\left(-u+N_{1}^{(n s)} u^{2}\right) t,
\end{aligned}
$$

and for the remainder of this section we let $Q_{2}=u^{2} t^{2} Q$.

When char $k \neq 2$, one can make $Q_{2}$ more explicit by calculating

$$
N_{1}^{(n s)}=\left\{\begin{array}{lll}
q-1 & \text { if } & \bar{b}_{2} \in k^{2} \\
q+1 & \text { if } & \bar{b}_{2} \notin k^{2}
\end{array}\right.
$$

and further note that $N_{1}^{(n s)}=N_{0}$ in this case. When char $k=2$, we shall leave $Q_{2}$ in terms of $N_{1}^{(n s)}$. With this notation the numerator in the $I_{2}$ case equals $P_{0}+Q_{2}$.

If $n>2, \bar{f}_{1}$ is singular. Again assuming the singularity is at the origin we have $\pi\left|a_{3,1}, \pi\right| a_{4,1}$ and $\pi \mid a_{6,2}$. Thus

$$
\bar{f}_{1}=y_{1}^{2}+\bar{a}_{1} x_{1} y_{1}-\bar{a}_{2} x_{1}^{2}
$$

where we note that the possible change of coordinates leaves $\bar{a}_{1}, \bar{a}_{2}$ and hence also $\bar{b}_{2}$ invariant. As in Tate's algorithm let $P(T)=T^{2}+\bar{a}_{1} T-\bar{a}_{2}$. Then recalling that $\nu\left(b_{2}\right)=0$, we have $P(T)=0$ has 0 or 2 solutions in $k$ depending on whether or not $\bar{b}_{2} \in k^{2}$. Thus

$$
N_{1}=\left\{\begin{array}{lll}
2 q-1=2 u^{-1}-1 & \text { if } \bar{b}_{2} \in k^{2}, \\
1 & \text { if } \bar{b}_{2} \notin k^{2},
\end{array}\right.
$$

and two applications of the stationary phase formula give

$$
\begin{gathered}
Z(u, t)=P_{0} /(1-u t)+u^{2} t^{2} \tilde{I}_{1}, \\
\tilde{I}_{1}=P_{1} /(1-u t)+u^{2} t^{k_{1}} \tilde{I}_{2},
\end{gathered}
$$

with

$$
P_{1}=\left(1-N_{1} u^{2}\right)+\left(-u+\left(N_{1}-1\right) u^{2}+u^{3}\right) t .
$$

If $\nu\left(a_{6,2}\right)=1$, then $k_{1}=1, \bar{f}_{2}=-a_{6,3}$, and $\tilde{I}_{2}=1$. With our current assumptions, $\nu\left(a_{6,2}\right)=1$ is equivalent to $\nu\left(a_{6}\right)=\nu\left(b_{8}\right)=3$, which in turn is equivalent to $\nu(\Delta)=3$. Thus we are in the $I_{3}$ case. We have $Q=(1-u t)$ and the following expression for the numerator of the zeta function

$$
P_{0}+u^{2} t^{2}\left(P_{1}+Q_{1}\right)
$$


If $n>3$, then $\nu\left(a_{6,2}\right)>1$ and $k_{1}=2$. If $\bar{f}_{2}$ is nonsingular, then $\nu\left(b_{8}^{(2)}\right)=0$, but this is equivalent to $\nu\left(b_{8}\right)=\nu(\Delta)=4$. Now $\bar{f}_{2}$ has the same form as $\bar{f}_{1}^{(n s)}$ so $N_{2}=N_{1}^{(n s)}$. Thus in the $I_{4}$ case the numerator of the zeta function is

$$
P_{0}+u^{2} t^{2}\left(P_{1}+Q_{2}\right) \text {. }
$$

In the $n>4$ case, $k_{1}=2, \bar{f}_{2}$ is singular, and the process continues.

For the general $I_{n}$ case, we let $m=[(n-1) / 2]$ and write $n=2 m+l$ with $l=1$ or $l=2$ with $n$ being odd or even, respectively. Then the $I_{n}$ case is determined after $m+1$ iterations of the stationary phase formula. For $1 \leq i \leq m$ we have $\bar{f}_{i}=y_{i}^{2}+\bar{a}_{1} x_{i} y_{i}-\bar{a}_{2} x_{i}^{2}$ with our original $\bar{a}_{1}$ and $\bar{a}_{2}$; hence, $N_{i}=N_{1}$ and $P_{i}=P_{1}$ for $0 \leq i \leq m$. Thus the application of the stationary phase formula to $\tilde{I}_{i}$ gives a contribution of $u^{2 i} t^{2 i} P_{1}$ to the numerator. If $n$ is odd we get $\bar{f}_{m+1}=-\bar{a}_{6, n}$ with $\nu\left(a_{6}\right)=\nu(\Delta)=n$. Thus the final contribution to the numerator is $u^{2 m} t^{2 m} Q_{1}$. If $n$ is even we get $\bar{f}_{m+1}$ is nonsingular since $\nu\left(b_{8}^{(m+1)}\right)=0$ which implies $\nu\left(b_{8}\right)=$ $\nu(\Delta)=2 m+2=n$. We have $N_{m+1}=N_{1}^{(n s)}$ and thus the final contribution is $u^{2 m} t^{2 m} Q_{2}$.

Then from the above description we obtain

Theorem 3.1. The numerator of the Igusa local zeta function associated to an elliptic curve $f(x, y)=0$ in the $I_{n}$ case for $n \geq 1$ is

$$
P_{0}+\left(u^{2} t^{2}+u^{4} t^{4}+\cdots+u^{2 m} t^{2 m}\right) P_{1}+u^{2 m} t^{2 m} Q_{l},
$$

where $n=2 m+l$ and we recall that

$$
\begin{aligned}
& P_{1}=\left(1-N_{1} u^{2}\right)+\left(-u+\left(N_{1}-1\right) u^{2}+u^{3}\right) t, \\
& Q_{l}= \begin{cases}u^{2} t-u^{3} t^{2} & \text { if } l=1, \\
u^{2}\left(1-N_{1}^{(n s)} u^{2}\right) t^{2}+u^{2}\left(-u+N_{1}^{(n s)} u^{2}\right) t^{3} & \text { if } l=2 .\end{cases}
\end{aligned}
$$

The above expression can be made more explicit by noting that when char $k \neq 2$,

$$
\begin{gathered}
N_{0}=N_{1}^{(n s)}=u^{-1}-1 \text { and } N_{1}=2 u^{-1}-1 \text { if } \bar{b}_{2} \in k^{2}, \\
N_{0}=N_{1}^{(n s)}=u^{-1}+1 \text { and } N_{1}=1 \text { if } \bar{b}_{2} \notin k^{2} .
\end{gathered}
$$

\section{The Determination of The local Zeta FunCtion For The CASES $I_{n}^{*}$.}

In the $I_{n}^{*}$ cases we can assume after a change of coordinates that $\pi$ divides $a_{1}$ and $a_{2}, \pi^{2}$ divides $a_{3}$ and $a_{4}$, and $\pi^{3}$ divides $a_{6}$. Two applications of the stationary phase formula give

$$
\begin{aligned}
Z(u, t) & =P_{0} /(1-u t)+u^{2} t^{2} \tilde{I}_{1}, \\
\tilde{I}_{1} & =P_{1} /(1-u t)+u t \tilde{I}_{2},
\end{aligned}
$$

with $N_{0}=q, S_{0}=1, N_{1}=S_{1}=q$; hence,

$$
\begin{aligned}
& P_{0}=(1-u)+\left(-u^{2}+u^{3}\right) t, \\
& P_{1}=(1-u)+\left(-u+u^{2}\right) t .
\end{aligned}
$$

We now have $-\bar{f}_{2}=x_{2}^{3}+\bar{a}_{2,1} x_{2}^{2}+\bar{a}_{4,2} x_{2}+\bar{a}_{6,3}$.

We first consider the $I_{0}^{*}$ case. In this case $\bar{f}_{2}$ has three distinct roots in $\bar{k}$, the algebraic closure of $k$. Hence $\bar{f}_{2}$ has either three distinct roots in $k$, one root in $k$, or 
no roots in $k$. We then get $\tilde{I}_{2}=Q_{2} /(1-u t)$ with $Q_{2}=\left(1-N_{2} u^{2}\right)+\left(-u+N_{2} u^{2}\right) t$. Thus, the numerator in the $I_{0}^{*}$ case is $P_{0}+u^{2} t^{2} P_{1}+u^{3} t^{3} Q_{2}$, which is equal to

$\mathrm{I}_{0}^{*}: \quad(1-u)+\left(-u^{2}+u^{3}\right) t+\left(u^{2}-u^{3}\right) t^{2}+\left(u^{4}-N_{2} u^{5}\right) t^{3}+\left(-u^{4}+N_{2} u^{5}\right) t^{4}$

with

$$
N_{2}= \begin{cases}3 q=3 u^{-1} & \text { if } \bar{f}_{2} \text { has } 3 \text { roots in } k, \\ q=u^{-1} & \text { if } \bar{f}_{2} \text { has } 1 \text { root in } k, \\ 0 & \text { if } \overline{f_{2}} \text { has no roots in } k .\end{cases}
$$

In the cases $I_{n}^{*}, n>0$, we have that $\bar{f}_{2}$ has one simple root and one double root in $\bar{k}$. By considering the derivative of $\bar{f}_{2}$, we conclude that the double root must be in $k$. We change coordinates so the double root is at the origin.

We apply the stationary phase formula again to get $\tilde{I}_{2}=P_{2} /(1-u t)+u t \tilde{I}_{3}$ with $N_{2}=2 q, S_{2}=q$; hence,

$$
P_{2}=(1-2 u)+u^{2} t
$$

When $\bar{f}_{3}=y_{3}^{2}+\bar{a}_{3,2} y_{3}-\bar{a}_{6,4}$ has distinct roots in $\bar{k}$, we are in the $I_{1}^{*}$ case. Hence, $\bar{f}_{3}$ has distinct roots in $k$ or no roots in $k$. Thus, in the $I_{1}^{*}$ case, we have $\tilde{I}_{3}=Q_{3} /(1-u t)$ with

$$
Q_{3}=\left(1-N_{3} u^{2}\right)+\left(-u+N_{3} u^{2}\right) t
$$

and the numerator in the $I_{1}^{*}$ case is equal to $P_{0}+u^{2} t^{2} P_{1}+u^{3} t^{3} P_{2}+u^{4} t^{4} Q_{3}$ with

$$
N_{3}= \begin{cases}2 u^{-1} & \text { if } \bar{f}_{3} \text { has } 2 \text { roots in } k \\ 0 & \text { if } \overline{f_{3}} \text { has no roots in } k .\end{cases}
$$

If $\bar{f}_{3}$ has a double root in $\bar{k}$, but not in $k$, the above applies with $N_{3}=0$.

If $\bar{f}_{3}$ has a double root in $k$ we apply the stationary phase formula again to get $\tilde{I}_{3}=P_{3} /(1-u t)+u t \tilde{I}_{4}$, where we note that $N_{3}=N_{1}$ and $S_{3}=S_{1}$, hence $P_{3}=P_{1}$. When $-\bar{f}_{4}=\bar{a}_{2,1} x_{4}^{2}+\bar{a}_{4,3} x_{4}+\bar{a}_{6,5}$ has distinct roots in $\bar{k}$ we are in the $I_{2}^{*}$ case. Thus, $\bar{f}_{4}$ has either distinct roots in $k$ or no roots in $k$. Then $\tilde{I}_{4}=Q_{4} /(1-u t)$ with

$$
Q_{4}=\left(1-N_{4} u^{2}\right)+\left(-u+N_{4} u^{2}\right) t,
$$

and thus we obtain that the numerator in the $I_{2}^{*}$ case is

$$
P_{0}+u^{2} t^{2} P_{1}+u^{3} t^{3} P_{2}+u^{4} t^{4} P_{1}+u^{5} t^{5} Q_{4}
$$

with

$$
N_{4}= \begin{cases}2 u^{-1} & \text { if } \bar{f}_{4} \text { has } 2 \text { roots in } k \\ 0 & \text { if } \bar{f}_{4} \text { has no roots in } k\end{cases}
$$

If $\bar{f}_{4}$ has a double root in $\bar{k}$ but not in $k$, the above applies with $N_{4}=0$.

If $\bar{f}_{4}$ has a double root in $k$, the process continues. For general $i$ we have $\tilde{I}_{i}=P_{i} /(1-u t)+u t \tilde{I}_{i+1}$, if $\bar{f}_{i}$ has a double root in $k$ or $\tilde{I}_{i}=Q_{i} /(1-u t)$ with

$$
Q_{i}=\left(1-N_{i} u^{2}\right)+\left(-u+N_{i} u^{2}\right) t
$$

and

$$
N_{i}=\left\{\begin{array}{lll}
2 u^{-1} & \text { if } & \bar{f}_{i} \text { has } 2 \text { roots in } k \\
0 & \text { if } & \bar{f}_{i} \text { has no roots in } k
\end{array}\right.
$$


The process terminates when

$$
\bar{f}_{n+2}= \begin{cases}-\left(\bar{a}_{2,1} x_{n+2}^{2}+\bar{a}_{4, \frac{n}{2}+2} x_{n+2}+\bar{a}_{6, n+3}\right) & \text { if } n \text { is even } \\ y_{n+2}^{2}+\bar{a}_{3, \frac{(n-1)}{2}+2} y_{n+2}-\bar{a}_{6, n+3} & \text { if } n \text { is odd }\end{cases}
$$

has distinct roots in $k$ or no roots in $k$, which eventually occurs and corresponds to the $I_{n}^{*}$ case of the Kodaira-Néron classification. The numerator for the $I_{n}^{*}$ case for $n \geq 2$ is

$$
P_{0}+u^{2} t^{2} P_{1}+u^{3} t^{3} P_{2}+\left(u^{4} t^{4}+u^{5} t^{5}+\cdots+u^{n+2} t^{n+2}\right) P_{1}+u^{n+3} t^{n+3} Q_{n+2}
$$

with

$$
N_{n+2}= \begin{cases}2 u^{-1} & \text { if } \bar{f}_{n+2} \text { has } 2 \text { roots in } k, \\ 0 & \text { if } \bar{f}_{n+2} \text { has no roots in } k .\end{cases}
$$

An inductive argument using the above formula shows

Theorem 4.1. The numerator of the Igusa local zeta function for an elliptic curve $f(x, y)$ in the $I_{n}^{*}$ case for $n \geq 1$ is

$$
(1-u)+\left(-u^{2}+u^{3}\right) t+\left(u^{2}-u^{3}\right) t^{2}-u^{4} t^{3}+u^{4} t^{4}-u^{n+4} t^{n+3}+u^{n+4} t^{n+4}
$$

when $\bar{f}_{n+2}$ has distinct roots, i.e., when

$$
\begin{gathered}
\overline{a c}\left(a_{4}^{2}-4 a_{2} a_{6}\right) \in k^{2} \text { if } n \text { is even, } \\
\overline{a c}\left(b_{6}\right) \in k^{2} \text { if } n \text { is odd }
\end{gathered}
$$

and

$$
(1-u)+\left(-u^{2}+u^{3}\right) t+\left(u^{2}-u^{3}\right) t^{2}-u^{4} t^{3}+u^{4} t^{4}+u^{n+4} t^{n+3}-u^{n+4} t^{n+4}
$$

when $\bar{f}_{n+2}$ has no roots, i.e., when

$$
\begin{gathered}
\overline{a c}\left(a_{4}^{2}-4 a_{2} a_{6}\right) \notin k^{2} \text { if } n \text { is even, } \\
\overline{a c}\left(b_{6}\right) \notin k^{2} \quad \text { if } n \text { is odd. }
\end{gathered}
$$

\section{The Numerators OF THE LOCAL ZETA FUnCTION} FOR ALL THE REMAINING CASES

ACCORDING TO THEIR KODAIRA-NÉRON CLASSIFICATION

The remaining cases are denoted II, III, IV, IV*, III*, II* and their numerators are as follows:

II

$$
1-u+u^{3} t-u^{3} t^{2}
$$

II*

$$
1-u+\left(-u^{2}+u^{3}\right) t+\left(u^{2}-u^{3}\right) t^{2}+u^{6} t^{5}-u^{6} t^{6},
$$

III, III*

$$
(1-u)\left(1-u^{2} t+u^{2} t^{2}\right) .
$$

Case IV:

IV a) $\quad 1-u+\left(-u^{2}+u^{3}\right) t+\left(u^{2}-2 u^{3}\right) t^{2}+u^{3} t^{3} \quad$ if $\overline{a c}\left(b_{6}\right) \in k^{2}$,

IV b) $\quad 1-u+\left(-u^{2}+u^{3}\right) t+u^{2} t^{2}-u^{3} t^{3}$ if $\overline{a c}\left(b_{6}\right) \notin k^{2}$.

Case IV*:

$\mathrm{IV}^{*}$ a) $\quad 1-u+\left(-u^{2}+u^{3}\right) t+\left(u^{2}-u^{3}\right) t^{2}-u^{5} t^{4}+u^{5} t^{5}$ if $\overline{a c}\left(b_{6}\right) \in k^{2}$,

$\mathrm{IV}^{*}$ b) $\quad 1-u+\left(-u^{2}+u^{3}\right) t+\left(u^{2}-u^{3}\right) t^{2}+u^{5} t^{4}-u^{5} t^{5}$ if $\overline{a c}\left(b_{6}\right) \notin k^{2}$. 


\section{REFERENCES}

[1] J.-I. Igusa, A stationary phase formula for p-adic integrals and its applications, Algebraic geometry and its applications, Springer-Verlag, (1994), 175-194. MR 95a:11104

[2] D. Meuser, On the poles of a local zeta function for curves, Invent. Math. 73 (1983), 445-465. MR 85i:14014

[3] J. Silverman, Advanced topics in the arithmetic of elliptic curves, Graduate Texts in Mathematics 151, Springer-Verlag (1994). MR 96b:11074

[4] J. Tate, Algorithm for determining the type of a singular fiber in an elliptic pencil, Modular functions of one variable IV, Lecture Notes in Math. 476, B.J. Birch and W. Kuyk, eds., Springer-Verlag, Berlin (1975), 33-52. MR 52:13850

[5] W. Veys, On the poles of Igusa's local zeta function for curves, J. London Math. Soc. 41 (1990), 27-32. MR 92j:11142

Boston University, Boston, Massachusetts 02215

E-mail address: dmm@math.bu.edu

Mount Holyoke College, South Hadley, Massachusetts 01075

E-mail address: robinson@mtholyoke.edu 